\title{
ON THE RATE OF STRONG CONVERGENCE FOR A RECURSIVE PROBABILITY DENSITY ESTIMATOR OF END SAMPLES AND ITS APPLICATIONS
}

\begin{abstract}
YONGMING LI
Abstract. The purpose of this paper is to consider a kind of recursive density estimator of the probability density function for a sequence of extended negatively dependent random variables. Under some suitable conditions, we establish the strong convergence rate for the recursive density estimator. As application, we discuss the strong convergence rate for a kind of hazard rate function estimator.
\end{abstract}

Mathematics subject classification (2010): 62G05, 62G20.

Keywords and phrases: END sequence, recursive density estimator, Hazard rate function, Strong convergence rate.

\section{REFERENCES}

[1] L. Liu, Precise large deviations for dependent random variables with heavy tails, Stat. Probabil. Lett, 2009, 79 (9): 1290-1298.

[2] Y. Q. Chen, A. Y. Chen, W. NG KAI, The strong law of large numbers for extended negatively dependent random variables, J. Appl. Prob., 2010, 47 (4): 908-922.

[3] L. LIU, Necessary and sufficient conditions for moderate deviations of dependent random variables with heavy tails, Sci. China Ser. A: Math. 2010, 53 (6): 1421-1434.

[4] A. T. SHEN, Probability inequalities for END sequence and their applications, J. Inequal. Appl., 2011, 2011 (98): 1-13.

[5] Y. F. WU, M. GuAN, Convergence properties of the partial sums for sequences of end random variables, J. Korean Math. Soc. 2012, 49 (6): 1097-1110.

[6] X. J. Wang, T. C. Hu, A. Volodin, S. H. Hu, Complete convergence for weighted sums and arrays of rowwise extended negatively dependent random variables, Communications in StatisticsTheory and Methods, 2013, 42 (13): 2391-2401.

[7] X. J. Wang, L. L. Zheng, C. XU, S. H. Hu, Complete consistency for the estimator of nonparametric regression models based on extended negatively dependent errors, Statistics: A Journal of Theoretical and Applied Statistics, 2015, 49 (2): 396-407.

[8] C. T. Wolverton, T. J. WAGNer, Asymptotically optimal discriminant functions for pattern classification, IEEE Trans Inform Theory, 1969, 15: 258-265.

[9] H. Y. LIANG, J. BAEK, Asymptotic normality of recursive density estimates under some dependence assumptions, Metrika, 2004, 60: 155-166.

[10] Y. M. LI, S. C. YANG, Strong convergence rate of recursive probability density estimator for NA sequences, Chinese J. Engineering Mathematics, 2005, 22 (4): 659-665.

[11] Y. M. LI, C. D. WeI, S. C. YANG, The recursive kernel distribution function estimator based on negatively and positively associated sequences, Communications in Statistics-Theory and Methods, 2010, 39 (20): 3585-3595.

[12] S. C. YANG, Consistency of nearest neighbor estimator of density function for negative associated samples, Acta Mathematice applicatae Sinica, 2003, 26 (3): 385-395. 